\title{
THE ELASTIC MODULI OF HUMAN SUBCHONDRAL, TRABECULAR, AND CORTICAL BONE TISSUE AND THE SIZE-DEPENDENCY OF CORTICAL BONE MODULUS*
}

\author{
K. Choi, J. L. Kuhn, M. J. Ciarelli and S. A. Goldstein $\dagger$ \\ The Biomechanics, Trauma, and Sports Medicine Laboratory. The University of Michigan, \\ Section of Orthopaedics, Department of Surgery, and the Bioengineering Program, Ann Arbor. \\ Michigan, U.S.A.
}

\begin{abstract}
A batract - The elastic moduli of human subchondral, trabecular, and cortical bone tissue from a proximal tibia were experimentally determined using three-point bending tests on a microstructural level. The mean modulus of subchondral specimens was $1.15 \mathrm{GPa}$, and those of trabecular and cortical specimens was $4.59 \mathrm{GPa}$ and $5.44 \mathrm{GPa}$ respectively. Significant differences were found in the modulus values between bone tissues, which may have mainly resulted from the differences in the microstructures of each bone tissue rather than in the mineral density. Furthermore. the size-dependency of the modulus was examined using eight different sizes of cortical specimens (heights $h=100-1000 \mu \mathrm{m}$ ). While the modulus values for relatively large specimens $(h>500 \mu \mathrm{m})$ remained fairly constant (approximately $15 \mathrm{GPa}$ ). the values decreased as the specimens became smaller. A significanl correlation was found between the modulus and specimen size. The surface area to volume ratio proved to be a key variable to explain the size-dependency.
\end{abstract}

\section{INTRODUCTION}

Determining the elastic modulus of bone tissuc on a microstructural level is important for understanding the mechanical behavior and function of bone. While several investigators have reported or predicted varying moduli for different size specimens of cortical bone, little data exist on subchondral tissue, and conflicting data have been reported on trabecular tissue. In the past, many structural models of bones or joints have assumed that all bone tissues possess the same modulus values, those of large cortical bone specimens (13-17 GPa) regardless of their type or size. This might lead to a misinterpretation of the structural functions of each bone tissue type as well as their role in the mechanical behavior of normal and implanted joints.

To the best of our knowledge, no modulus measurements have been made on microspecimens of subchondral tissue. However, relatively large specimens have been tested, and the moduli have ranged from approximately 1 to $3 \mathrm{GPa}$; considerably lower than the previously assumed values (Murray et al., 1984; Brown and Vrahas, 1984). The reported trabecular tissue moduli from microspecimens have ranged from approximately 1 to $13 \mathrm{GPa}$ (Runkle and Pugh, 1975; Townsend et al., 1975; Williams and Lewis, 1982; Ryan and Williams, 1989; Mente and Lewis, 1987; Ashman and Rho, 1988; Rice et al., 1988; Kuhn et al., 1989). While this wide range could be explained by the

Received in final form 20 April 1990.

- Presented in part at the 35th Annual Meeting of the Orthopaedic Research Society, Las Vegas, February 1989.

t Reprint requests to: Steven A. Goldstein. Ph.D.. Biomechanics Laboratory, G-0161 400 N. Ingalls, Ann Arbor. MI $48109-0486$, U.S.A. differences in testing methods and materials, the nonmachined and irregularly shaped specimens used in some studies (Runkle and Pugh, 1975; Townsend et al., 1975; Ryan and Williams, 1989) may have played an important role.

In a previous study in our laboratory, Kuhn and coworkers (1989) were able to produce regularly shaped specimens from human iliac crest trabecular and cortical tissues on the microstructural level, and reported the mean tissue moduli to be $3.71 \mathrm{GPa}$ and 4.77 GPa, respectively. These values were significantly lower than reported values for cortical bone, and suggested that specimen size effect may be very important at the microstructural level. Low moduli for single osteons have been reported in a series of works by Ascenzi and Bonucci $(1967,1968)$. An apparent size-dependency effect on modulus was also found in tests on cortical specimens by Murray et al. (1984). Recently, Rice et al. (1988) proposed that the higher surface area to volume ratio in microspecimens might lower the modulus due to the 'surface imperfections' in bone specimens. However, this speculation remains to be evaluated.

The purpose of this study was (1) to experimentally determine the elastic moduli of subchondral, trabecular, and cortical bone tissues on a microstructural level using regular rectangular beam specimens from a human tibia and (2) to investigate the size-dependency of the modulus using eight different sizes of cortical bone specimens.

\section{MATERIAIS}

Two different sets of specimens were prepared for mechanical tests: (1) microspecimens for the determination of the elastic moduli of the bone tissues and 
Table 1. The mean modulus and mineral density values for each type of bone specimen. All specimen dimensions are reported in units of microns, the modulus values in units of $\mathrm{GPa}_{\text {. }}$ and the mineral density in units of $U$ (grey scale/area). Standard deviations are shown in parentheses

\begin{tabular}{lccccc}
\hline Bone type & $n$ & Height & Base & $\begin{array}{c}\text { Elastic } \\
\text { modulus }\end{array}$ & $\begin{array}{c}\text { Mineral density } \\
\text { measurements }\end{array}$ \\
\hline Subchondral bone & 20 & 144.2 & 156.6 & $1.15(0.37)$ & $0.36(0.04)$ \\
$\begin{array}{c}\text { Trabecular } \\
\text { bone }\end{array} \quad\left[\begin{array}{llll}\text { vertical } \\
\text { horizontal } \\
\text { total }\end{array}\right.$ & 13 & 126.6 & 119.7 & $4.87(1.84)$ & $0.36(0.05)$ \\
Cortical bone & 20 & 127.9 & 116.9 & $3.83(0.45)$ & $0.32(0.03)$ \\
& 17 & 129.3 & 124.9 & $5.44(1.25)$ & $0.29(0.03)$ \\
\hline
\end{tabular}

(2) various sizes of cortical bone specimens for the sizedependency study. All bone specimens were obtained from one human right tibia $(60$, healthy male, death by a sudden heart attack) in order to avoid the effects of different donors on the modulus. The tibia was obtained within $72 \mathrm{~h}$ of death, and stored at $-10^{\circ} \mathrm{C}$.

\section{METHODS}

\section{Specimen preparation}

Microspecimens. Both trabecular and subchondral specimens were obtained from the medial side of the proximal tibia (top $8 \mathrm{~mm}$ ), while cortical specimens were obtained from the posterior aspect of the tibial diaphysis. Specimens were prepared by using similar procedures previously described by Kuhn et al. (1989). A series of bone sections $(100-170 \mu \mathrm{m})$ were cut on a low-speed diamond blade saw (Model $11-1180$ Isomet. Buehler, Lake Bluff, IL 60044) under constant irrigation. Trabecular bone sections were cleared of marrow by using a needle so that all voids were delineated, and relatively continuous segments were cut from the sections using a scalpel blade. Similar segments were cut from subchondral and cortical bone sections as a preliminary step in the microspecimen preparation. Since the sectioning process produced two parallel surfaces on the bone segments, it was only necessary to make the other surfaces parallel to create rectangular beam specimens. A specially designed miniature milling machine was used for this purpose. This machine consisted of a specimen holding fixture attached to an $x-y$ table (Model 4464, Daedal Inc., Harrison City, PA 15636), and a high-speed grinder (Model 604, Federal-Mogul Corporation, Chicago, IL. 60646) with a miniature drill bit of $200 \mu \mathrm{m}$ diameter (No. 3143, Vern Wheeler and Company, Livonia, MI 48151). The vertical and horizontal movements of the $x-y$ table were controlled by precision micrometers. Each bone segment was clamped into the holding fixture and milled to a thickness of $100-170 \mu \mathrm{m}$ under constant irrigation. Thus, rectangular beam specimens $\left\{(100-170) \times(100-170) \times(\sim 1500) \mu \mathrm{m}^{3}\right\}$ were produced from each bone tissue.

Trabecular specimens were machined from both vertically (superior-inferior, S-I direction) and hori- zontally (medial-lateral, M-L direction) oriented trabeculae. Subchondral specimens were machined from the most proximal portion of the subchondral plate (50-300 $\mu \mathrm{m}$ below tidemark), and the long axis of each subchondral specimen was oriented in an $M-L$ direction. The long axis of each cortical specimen was oriented in an S-I direction. Most of the procedures were conducted under a stereomicroscope at $40 x$ magnification.

Cortical bone specimens for size-dependency study. Additional cortical bone specimens for the size-dependency study were obtained from the posterior aspect of the tibial diaphysis, and prepared using only the low-speed saw. Sections with varying thickness (approximately 100-1000 $\mu \mathrm{m}$ ) were cut on the saw. Each cortical bone section was then placed between two plates of plexiglass and cut sequentially, thus all four surfaces of rectangular beam specimens were produced by the saw. Eight different sizes of cortical bone specimens were prepared.

\section{Measurements of specimen dimensions}

The specimen dimensions (height and base) were measured by using a digital image processing system (Recognition Technology Inc., Westborough, MA 01581) hosted by an IBM PC computer. Each specimen was observed through a light microscope and imaged with a video camera (Series 68, Dage-MTI Inc., Michigan City, IN 46360). The dimensions were determined by digitizing the image at a magnification of $0.95-9.5 \mu \mathrm{m} / \mathrm{pixel}$ depending on the specimen size. All specimens were kept moist during the whole procedure. Specimens with height to base ratios greater than 1.5 or smaller than $1 / 1.5$ were excluded from this study.

A total of 57 microspecimens (20 subchondral, 20 trabecular, and 17 cortical specimens) and 88 cortical specimens (see Table 2) were prepared for the mechanical tests. Specimens were kept moist at all times and stored at $-10^{\circ} \mathrm{C}$ until testing.

\section{Mechanical tests}

A three-point bending test apparatus, shown in Fig. l, was used for mechanical tests (Kuhn et al., 1989). An 
Table 2. The modulus values for each specimen size group. All specimen dimensions are reported in units of microns, and the modulus values in units of $\mathrm{GPa}$. Standard deviations are shown in parentheses

\begin{tabular}{llrrr}
\hline Size group & $n$ & Height & Base & Elastic modulus \\
\hline 1 & 10 & 1002.3 & 997.7 & $14.92(1.75)$ \\
2 & 10 & 798.8 & 806.2 & $16.31(1.43)$ \\
3 & 10 & 564.2 & 516.1 & $14.51(2.36)$ \\
4 & 10 & 322.6 & 306.7 & $11.62(1.10)$ \\
5 & 10 & 193.0 & 209.9 & $8.91(2.14)$ \\
6 & 10 & 143.9 & 150.4 & $7.44(1.50)$ \\
7 & 15 & 122.8 & 131.7 & $5.15(1.47)$ \\
8 & 13 & 102.3 & 111.3 & $5.45(1.53)$ \\
\hline
\end{tabular}

ordinary microscope stage was converted into the testing apparatus by the following modifications. A platform, providing end supports for the specimens, was placed on a custom-made load cell, and this unit was attached to the microscope stage. The vertical movement of the stage was controlled by a stepper motor connected to the fine focal adjustment mechanism of the microscope. A loading head was attached to the stationary part of the microscope. With the aid of a stereomicroscope, each specimen was placed across the end supports, and loaded at its midpoint at a constant displacement rate of $0.11 \mathrm{~mm} \mathrm{~s}^{-1}$. An effort was made to stop loading prior to specimen fracture in order to save all specimens for further analyses. The displacement wasimeasured by a linear variable difierential transformer (Model 100 MHR, Schaevitz, Engineering, Pensauken, NJ 08110), and the magnitude of load was measured by the load cell. Load and displacement data were acquired simultaneously by using an analog-to-digital converter and an IBM PC computer. The following equation for elastic bending of a beam, which accounts for the effects of shear stress on modulus, was used to calculate the modulus for each specimen (Timoshenko and Goodier, 1987).

$$
E=\frac{(P / y) L^{3}}{48 l}\left\{1+2.85(h / L)^{2}-0.84(h / L)^{3}\right\}
$$

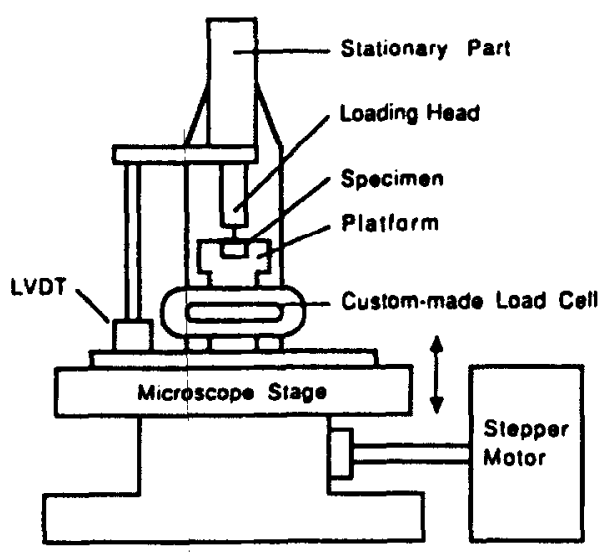

Fig. 1. Schematic diagram of the three-point bending test apparatus. where:

$E=$ elastic modulus in bending;

$P / y=$ initial slope of load-displacement curve:

$L=$ span length;

$I=$ area moment of inertia $\left[=\left\{(1 / 12) b h^{3}\right\}\right]$;

$b=$ base of the specimen;

$h=$ height of the specimen.

The aspect ratio of the beam specimens $(L / h)$ was kept constant (approximately 10) for both the microspecimens $(L=1.3, h=\sim 0.13 \mathrm{~mm})$ and various sizes of cortical specimens $(L=1.3-10.5, h=0.1-1.0 \mathrm{~mm})$. All calculated modulus values were corrected for the compliance of the testing system. All specimens were kept moist during the test.

\section{Measurements of mineralization}

An automated microradiographic technique, developed by Feldkamp and Jesion (1986), was used to determine relative differences in mineral density between microspecimens in a similar application used by Kuhn et al. (1989). This scanning system is mainly composed of an $X$-ray source, image intensifier, video camera, and digitizing system controlled by a VAX-730 computing system. A total of 54 specimens (18 subchondral, 20 trabecular, and 16 cortical specimens) were mounted on a platform and scanned sequentially. Note that three specimens (2 subchondral and 1 cortical) were lost during this procedure. The platform was placed as close as possible to the $X$ ray source to obtain a high magnification (331 pixels/mm). Images, captured by the intensifier, were transferred to the digitizing system through the camera. X-ray attenuation coefficients, assumed to be proportional to mineral density, were converted into grey scale values (256 levels). Attenuation profiles were created over planar projections of each specimen from the grey scale values. The relative mineral density (unit $=$ grey scale $\mathrm{mm}^{-2}$, U) was computed by integrating each attenuation profile and dividing by the specimen cross-sectional area. An average mineral density was determined from approximately 10 profiles taken at various locations along the length of each specimen. These measures represented relative differences in mineral density between specimens, which should be sufficient to investigate the effects of mineral density on modulus.

\section{RESULTS}

Validation tests for the specimen preparation technique and testing methods

One of our concerns was that the preparation technique might be creating artifactual defects on the surfaces of the specimens, thus affecting the modulus values. In order to evaluate the surface condition, both milled specimens and the specimens prepared using only the low-speed saw were immersed in $1 \%$ basic fuchsin in a $40 \%$ ethyl alcohol solution for a period of 
seven days, as described by Frost (1960). Nearly all the physiologic spaces and defects were stained. No artifactual defects due to the machining procedures were observed under high magnification [Fig. 2(a)]. Backscattered image analysis [Fig. 2(b)] through a scanning electron microscope (Hitachi S-750) also supported the observation of undamaged surfaces after machining. Although the possible artifactual defects due to the machining cannot be completely ruled out, we believe that the effects of the artifactual defects, if any, on the modulus might be negligible compared to those of biologic defects, such as lacunae $(5-30 \mu \mathrm{m})$.

The validity of the milling technique was further evaluated by comparison of the modulus values of the similar sized cortical specimens prepared by the two different techniques. The mean modulus of cortical specimens ( $h=123 \mu \mathrm{m}, n=15$ ) prepared using only the low-speed saw was 5.15 GPa (Table 2), while that of milled specimens $(h=129 \mu \mathrm{m}, n=17)$ was $5.44 \mathrm{GPa}$ (Table 1). Using a one-way ANOVA, no significant difference in modulus values was found between the two specimen groups $(p>0.05)$. This suggested that the surfaces produced by milling were as smooth as those produced by the low-speed saw and that the possible heating effects due to the high-speed milling procedure, which we were not able to evaluate directly, were no worse than those due to the standard low-speed sawing.

Since this study included both microspecimens and large specimens, the testing methodology was further evaluated by testing 20 circular microbeam specimens of silicon carbide fiber $(d=140 \mu \mathrm{m}$. Textron Specialty Materials, Lowell, MA 01851) and 10 acrylic rectangular beam specimens $(h=1 \mathrm{~mm})$. The measured mean modulus of the fiber specimens was $350.85 \mathrm{GPa}$ (s.d. $=17.84$ ), which was approximately $10 \%$ lower than a known range of values of 380-415 GPa provided by the manufacturer. The acrylic specimens tested provided a mean modulus value of $3.37 \mathrm{GPa}$ (s.d. $=0.07$ ), which was $8.7 \%$ higher than a known value of 3.10 GPa. Both differences were within $\pm 10 \%$, which we believed to be a reasonable level of accuracy considering the difierences between the manufacturer's and our testing methods.

The elastic moduli of subchondral, trabecular, and cortical bone tissue

Figure 3 illustrates an example of load-displacement curves generated by the testing apparatus. The initial slopes of the curves were used to calculate the elastic modulus values. A summary of the test results is presented in Table 1 . The modulus for subchondral specimens $(n=20)$ ranged from 0.62 to $1.88 \mathrm{GPa}$, with a mean value of $1.15 \mathrm{GPa}$ (s.d. $=0.37$ ). The modulus for trabecular specimens $(n=20)$ ranged from 3.27 to $10.58 \mathrm{GPa}$, with a mean value of $4.59 \mathrm{GPa}($ s.d. $=1.60)$. The modulus for cortical specimens $(n=17)$ ranged from 3.07 to $7.63 \mathrm{GPa}$, with a mean value of $5.44 \mathrm{GPa}$ (s.d. $=1.25$ ). An analysis of variance demonstrated

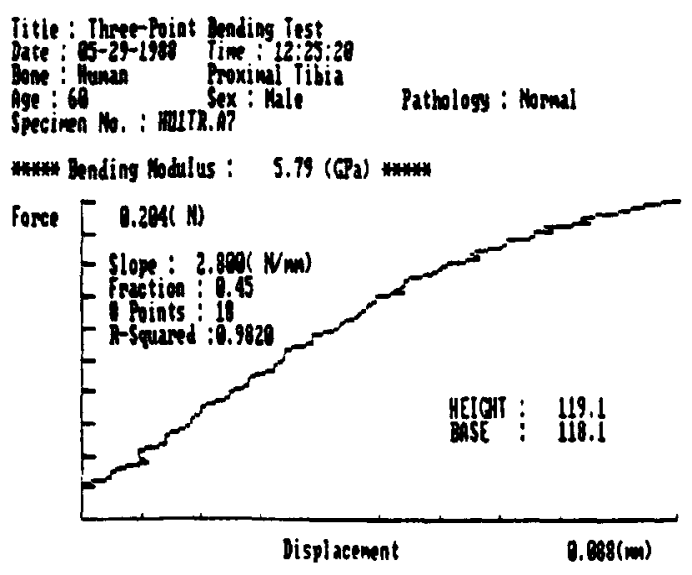

Fig. 3. A load-displacement curve for a trabecular specimen generated by the testing apparatus.

significant differences in the moduli between all bone tissues $(p<0.05)$. The mean modulus of cortical specimens was $19 \%$ higher than that of trabecular specimens.

The moduli of the vertically and horizontally oriented trabecular specimens were compared using a one-way ANOVA. Although the mean modulus of vertically oriented trabecular specimens $(n=13, E$ $=4.96 \mathrm{GPa}$, s.d. $=1.85$ ) was $27.5 \%$ higher than that of horizontally oriented specimens $(n=7, E=3.89 \mathrm{GPa}$, s.d. $=0.46$ ), no statistically significant difference was found. Also, no significant difference in the relative mineral density values was found between the vertically $(0.36 \mathrm{U}$, s.d. $=0.06)$ and horizontally $(0.32 \mathrm{U}$, s.d. $=0.03$ ) oriented trabecular specimens.

The mineral density values for subchondral specimens ranged from 0.31 to $0.44 \mathrm{U}$, with a mean value of $0.36 \mathrm{U}$ (s.d. $=0.04$ ). The values for trabecular specimens ranged from 0.25 to $0.50 \mathrm{U}$, with a mean value of $0.35 \mathrm{U}$ (s.d. $=0.05$ ). The values for cortical specimens ranged from 0.24 to $0.34 \mathrm{U}$, with a mean value of $0.29 \mathrm{U}$ (s.d. $=0.03$ ). These data showed that both the subchondral and trabecular specimens possessed significantly more mineral than the cortical specimens $(p<0.01)$. However, no significant difference between the subchondral and trabecular specimens was found.

Figure 4 illustrates linear (a) and logarithmic (b) regressions of the modulus vs the mineral density for each type of bone specimen. While statistically significant relationships $(p<0.01)$ were found in the trabecular specimens, no relationships were found in subchondral specimens. For cortical bone, the relationship was significant $(p<0.05)$ in the linear model, but not significant $(p=0.0525)$ in the logarithmic model.

\section{The size-dependency of cortical bone modulus}

The results of the study on the size effect on cortical bone modulus are demonstrated in Table 2 . The mean moduli of large specimens with heights greater than $550 \mu \mathrm{m}$ remained fairly constant, and they ranged 


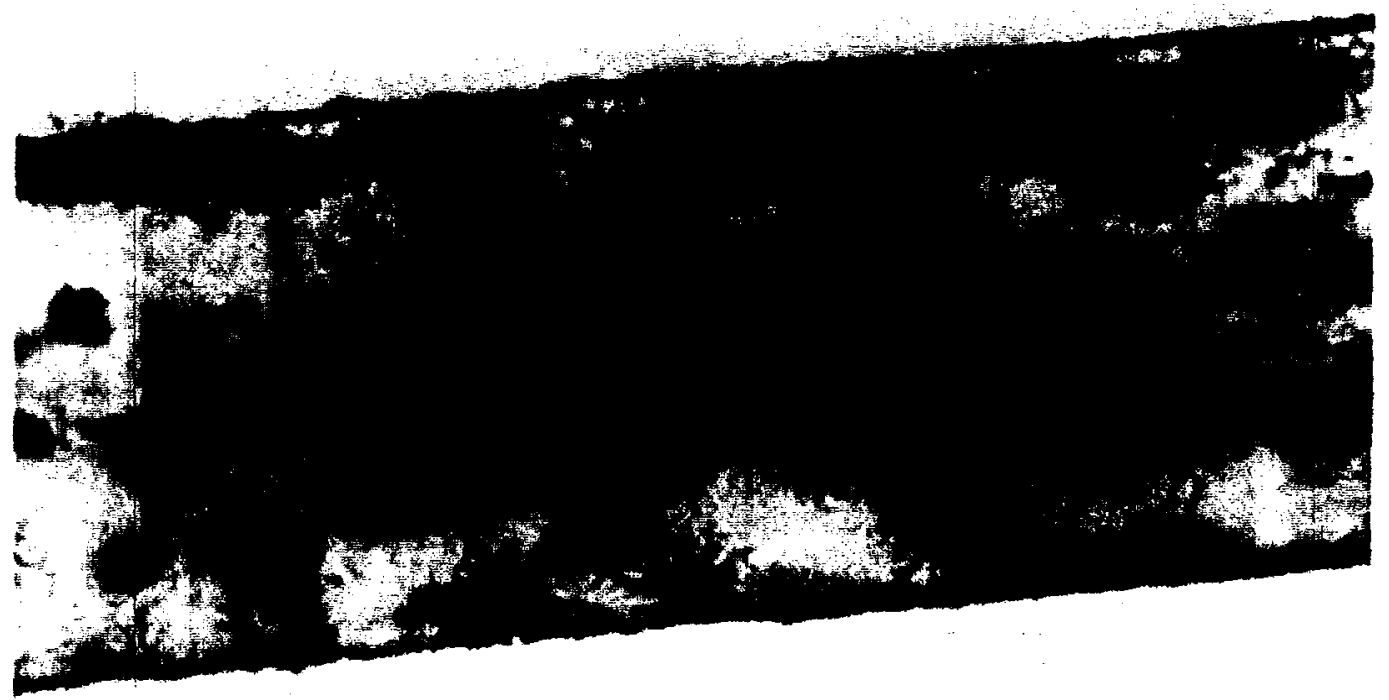

(a)

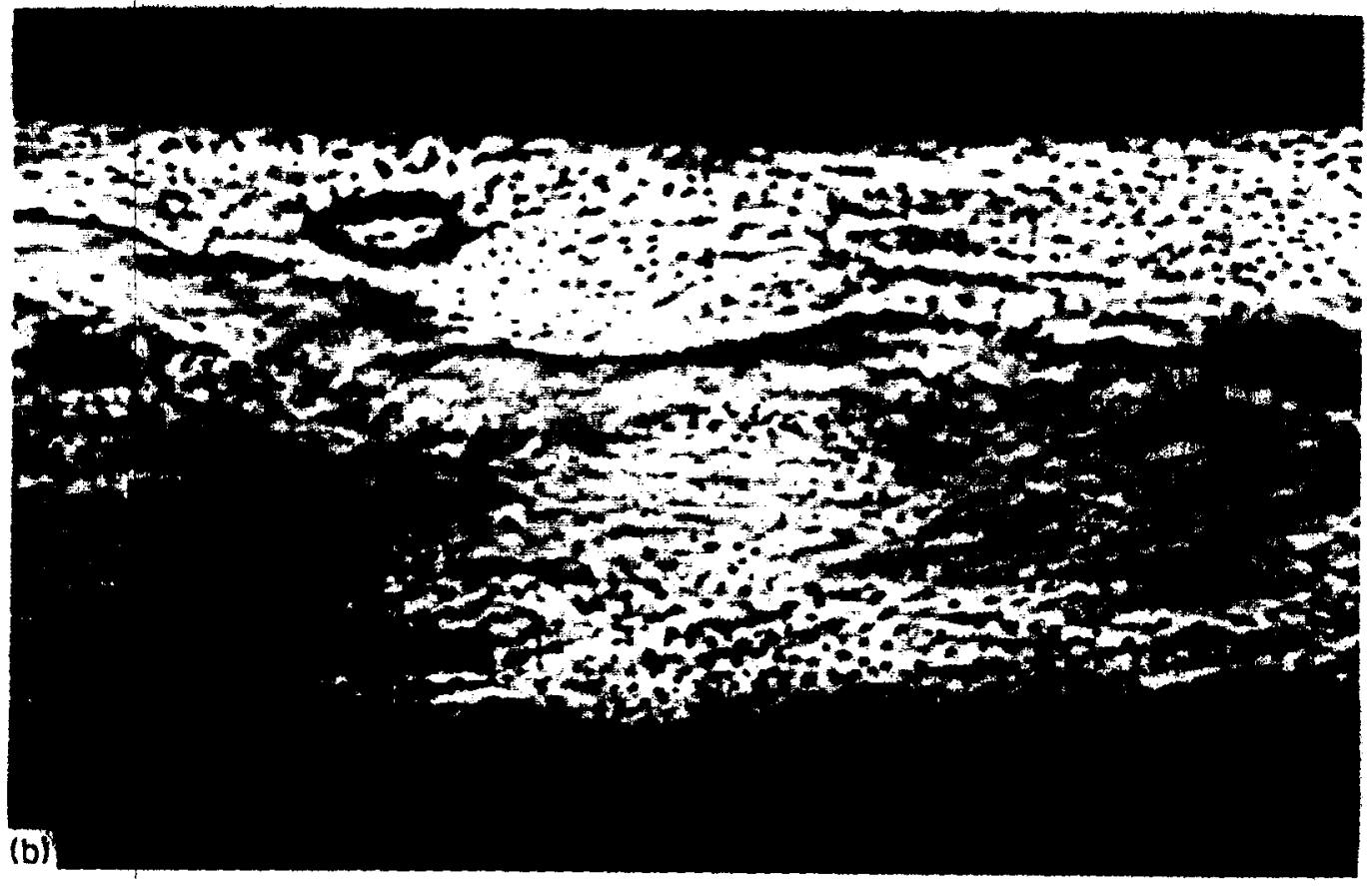

Fig. 2. (a) Purtions of a cortical microspecimen as viewed by urdinary light microscope (Isomet cut and stainad with $1 \%$ basic fuchsin); (b) backscattered electron image of a trabecular microspecimen (milled. tested. carbon-coated). Note the relative size of lacunate $(5-30 \mu \mathrm{m})$ compared to the specimen thickness (approximulcly $100 \mu \mathrm{m}$ ) 


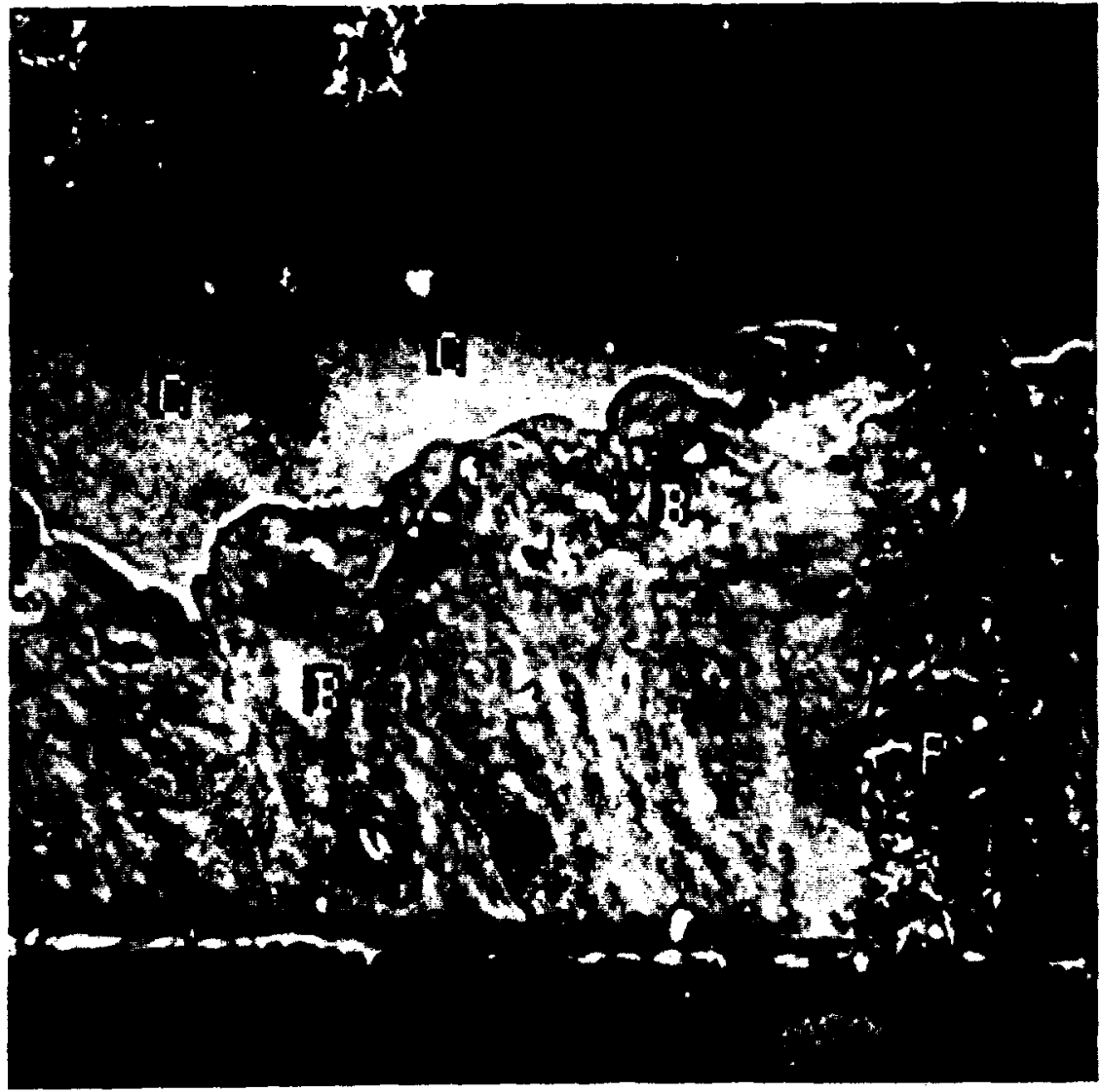

Fig. 6. Backscattered electron image of a subchondral microspecimen (milled, tested, carbon-coated), which shows that the specimen consists of calcified cartilage (C, brighter, higher mineral density) and bone tissue (B. darker, lower mineral density). Note also a perforation (P) within the specimen. 

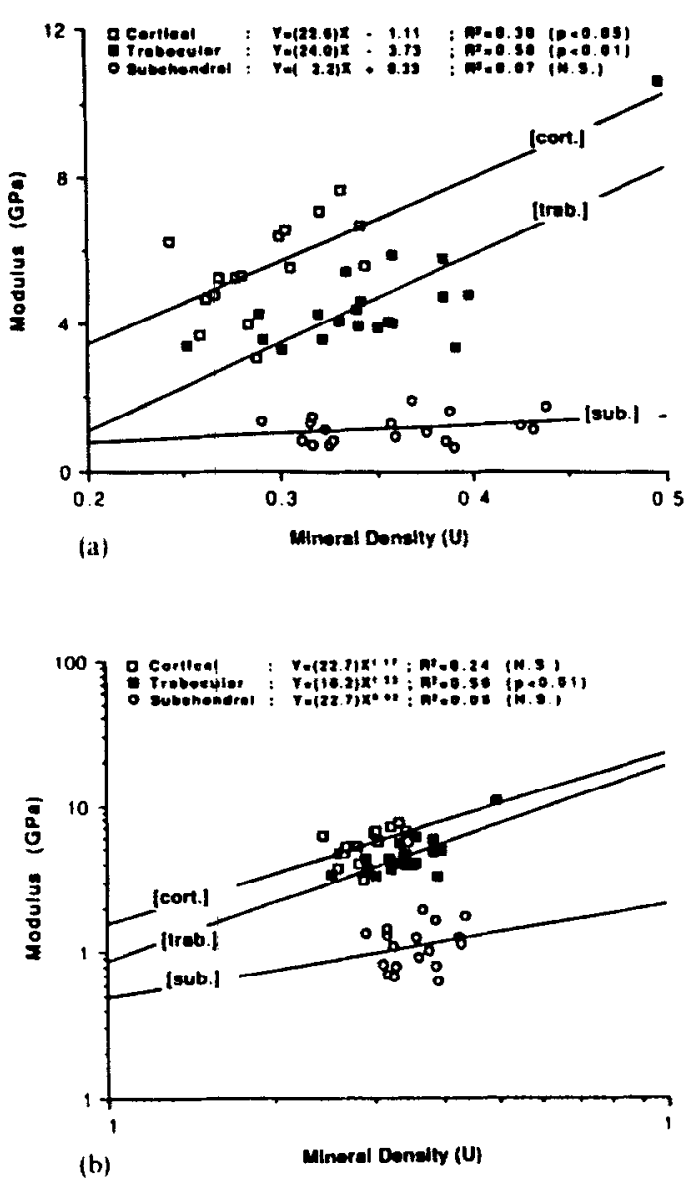

Fig. 4. Lincar (a) and logarithmic (b) regression models show correlations between the modulus and mineral density values for each type of bone specimen. While significant correlations were found in trabecular specimens, no significant correlations were found in subchondral specimens in both regressions. For cortical specimens, a significant correlation was found in the linear model, but not in the

logarithmic model. N.S. $=$ not significant $(p>0.05)$.

from 14.51 to $16.31 \mathrm{GPa}$. For the specimens with heights between 550 and $100 \mu \mathrm{m}$, the moduli decreased from 14.51 to $5.45 \mathrm{GPa}$, as the specimen size decreased. A significant relationship between modulus and specimen size was found $(p<0.01)$. Similarly, a significant relationship between modulus and surface area to volume ratio of specimens was also found (Fig. $5, p<0.01$ ). The data were fit to the following equation

$$
E_{\mathrm{co}}=A-B\left(S_{\mathrm{v}}\right)+C\left(S_{\mathrm{v}}\right)^{2}
$$

where:

$E_{\mathrm{co}}=$ elastic modulus of cortical specimen in bending:

$A, B, C=$ empirical constants: $A=18.34 \mathrm{GPa} ; \quad B$ $=613.08 \mathrm{GPa}(\mu \mathrm{m}): C=6961.5 \mathrm{GPa}(\mu \mathrm{m})^{2}$;

$S_{v}=$ surface area to volume ratio.

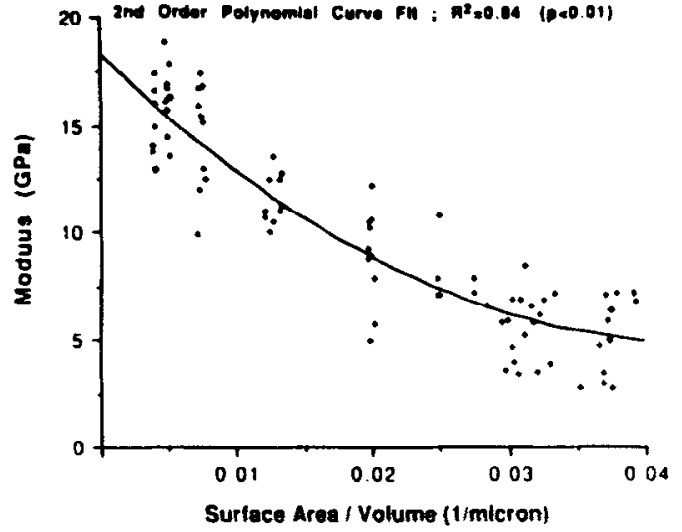

Fig. 5. The size-dependency of the cortical modulus is clearly shown by the strong correlations between modulus and surface area to volume ratio.

\section{Discussion}

Some considerations on specimen preparation and three-point hending methods

The effect of specimen preparation artifict on the modulus was evaluated by examining the specimen surface conditions utilizing a staining technique and scanning electron microscopy. While no artifactual defects were observed under high magnification, the possible defects due to machining cannot be completely ruled out. However, we believe that biologic defects such as lacunac more greatly affected the measured modulus than the possible defects caused by the machining process.

Three-point bending was chosen as the mechanical testing method, since the possible error due to end effects and specimen holding problems involved in tensile or compressive testing on microspecimens could be eliminated. However, the choice of threepoint bending raised several concerns which need to be addressed.

The main difficulty in the bending tests is the estimation of accurate stresses and strains from the load and displacement measurements. As discussed previously by Reilly and Burstein (1974), the estimation of actual strain based on cross-head displacement might introduce significant errors. A local stress perturbation due to the concentraled loading (shear stress effect) might also introduce some errors. The calculations in this study were corrected for the shear effect by equation (1) (Timoshenko and Goodier, 1987). While it is difficult to accurately estimate the total error due to the use of a bending test, the validation tests of silicon carbide fibers and acrylic specimens showed that the errors in determining the elastic modulus could be less than $10 \%$ for the isotropic and homogeneous materials.

The simple application of equation (1), which assumes isotropic materials with a Poisson's ratio of 0.3 , to anisotropic and heterogeneous materials like bone 
might introduce additional error sources (Tarnopol'skii and Kincis, 1985). The complex nature of the bone structure might cause nonlinear stress distributions due to shifting of the neutral axis, interlamellar shear stresses and stress concentrations around biologic defects. Since equation (1) does not consider nonlinear stress distributions over the width of the specimen, nonuniformity of specimen dimensions might also introduce errors. It is almost impossible to accurately estimate the total error due to anisotropy and heterogeneity, since there is no complete anisotropic bending theory, and the elastic symmetry of bone tissue is unknown. A recent investigation by Martin and Pramanik (1989), however, demonstrated that the Timoshenko correction seemed to account for the errors for bone specimens when the aspect ratio is greater than 10 . Their data also suggested that further correction is needed for thick and short specimens.

According to ASTM STPs applied to determine flexural moduli for plastic materials (ASTM D90-84), ceramic whiteware materials (ASTM C674-80), and adhesive bonded laminated assemblies (ASTM D1184-69), the recommended aspect ratios are 16,8, and 8, respectively. The ASTM tests also recommended the specimen height to base ratios to be $0.5-2.0$. The specimens used in this study had an aspect ratio of approximately 10 and a specimen height to base ratio range of $0.67-1.5$.

Table 3 shows some of the tensile and bending modulus values reported in the literature for wet human cortical bone specimens. These values demonstrated a small difference between tensile and bending moduli for human cortical bone, while Simkin and Robin (1973) reported a difference as great as $\mathbf{4 0} \%$ for bovine cortical bone. Our modulus values for large cortical specimens (15.2 GPa) agreed well with other modulus values, although it is not clear how much error the bending method introduced for microspecimens. A new theory for composite materials which accounts for the ultrastructure of bone would be necessary for a more accurate analysis. Lastly, despite the many limitations in a bending test, the past literature in bone mechanics lends some support for the test.

The elastic moduli of subchondral, trabecular, and cortical bone tissue

The subchondral specimens demonstrated a mean modulus value $(1.15 \mathrm{GPa})$, which was significantly lower than that of trabecular and cortical specimens in spite of the bigh mineral density. Since most of the specimens were obtained from the most proximal portion of the subchondral plate, with an average height of $144.2 \mu \mathrm{m}$, it might be reasonable to assume that the specimens were composed of both calcified cartilage and bone tissue. The high mineral density of subchondral specimens could be explained by the presence of the fully calcified cartilage in the specimens. Figure 6 shows a backscattered image of a subchondral specimen which consists of calcified cartilage and underlying bone. The presence of the cartilage was confirmed by staining the specimens with hematoxylin, fast green, and safranin-o.

The microstructural characteristics, along with the presence of calcified cartilage, might be responsible for the low modulus of subchondral specimens. According to the recent observations of Duncan et al. (1987). the subchondral bone plate contains numerous 'perforations' of various sizes, which are not seen in trabecular and cortical bone. Figure 6 illustrates such a perforation within a subchondral specimen. While the physiological function of the perforations is not entircly understood, their mechanical consequences are quite obvious. The microstructural defects will reduce the ability of subchondral bone to resist deformation, thus resulting in the low modulus.

Low modulus values of subchondral bone have also been reported in other studies. Murray et al. (1984) prepared thin disk-shaped subchondral bone specimens $(d=9.5 \mathrm{~mm}, t=0.5-0.9 \mathrm{~mm})$ from human proximal tibiae, applied a point-loading at the center of the disk, and found the mean modulus to be $3.06 \mathrm{GPa}$. Brown and Vrahas (1984) also conducted mechanical tests on thin shell-shaped femoral subchondral bone

Table 3. The comparison of the tensile and bending moduli from wet human cortical specimens. All moduli are reported in units of $\mathrm{GPa}$

\begin{tabular}{lrlll}
\hline Tensile modulus & \multicolumn{3}{c}{ Bending modulus } \\
\hline Evans and Lebow (1951) & 14.2 & Sedlin (1965) & 15.8 \\
Dempster and Liddicoat (1952) & 14.1 & Sedlin and Hirsch (1966) & 15.5 \\
Ko (1953) & 17.3 & Yamada (1970) & 15.7 \\
Sedlin and Hirsch (1966) & 6.0 & Currey and Butler (1975) & 15.7 \\
Yamada (1970) & 17.2 & Yang and Lakes (1982) & 14.4 \\
Burstein et al. (1972) & 14.1 & Present study & $15.2 \dagger$ \\
Reilly et al. (1974) & 17.9 & & \\
Reilly and Burstein (1975) & 17.0 & & 15.4 \\
Average & $16.0 \ddagger$ & Average & \\
\hline
\end{tabular}

- The value was obtained from the specimens over 40 yr old.

t The value was obtained from large specimens $(h>500 \mu \mathrm{m})$.

†The average value did not include Sedlin and Hirsch's data. 
specimens $(d=31.75 \mathrm{~mm})$ with various thickness values, and reported the apparent elastic modulus to be $1.37 \mathrm{GPa}$ for the specimens with a thickness of $1 \mathrm{~mm}$.

The trabecular specimens of this study demonstrated a mean modulus of $4.59 \mathrm{GPa}$, falling into the range of values $(1.0-13.0 \mathrm{GPa})$ previously reported by other investigators. Past studies have included tensile tests (1.0 GPa, Ryan and Williams, 1989), buckling tests (11.4 GPa, Townsend et al., 1975), ultrasonic evaluation (13.0 GPa, Ashman and Rho, 1988), finite element estimation $(1.3 \mathrm{GPa}$, Williams and Lewis, 1982), a combined cantilever bending and finite element analysis (5,3 GPa, Mente and Lewis, 1987), and three-point bending tests $(3.71 \mathrm{GPa}$, Kuhn et al., 1989).

In the present study, we found a mean trabecular tissue modulus to be $4.59 \mathrm{GPa}$ while a mean modulus of similar sized cortical specimens was $5.44 \mathrm{GPa}$. This showed that the cortical tissue modulus was $19 \%$ higher than the trabecular tissue modulus, and this difference was statistically significant $(p<0.05)$. This finding agreed well with the results in a previous study (Kuhn et al., 1989) where the difference was $28 \%$. While this difference has been previously predicted by other investigators in different manners, which include microscopic hardness lesting (Weaver, 1966; Hodgskinson et al., 1989) and predictions based on the difference in mineral density (Ashman and Rho, 1988), no direct data have been provided to explain the difference in moduli.

In the present study, the mineral density of trabecular specimens was higher than that of cortical specimens, while the trabecular tissue modulus was lower than the cortical tissue modulus. As described earlier, the same result was found in the subchondral bone specimens. This suggested that the estimation of the trabecular tissue modulus by extrapolating the modulus-mineral density ralationship for cortical bone may not be valid, and that microstructural differences between bone tissues play a critical role in determining the modulus on the microstructural level. Ascenzi and Bonucei $(1967,1968)$ demonstrated that even within cortical bone the moduli of single osteons are dependent on the mineral density as well as microstructure (types of osteons). A recent investigation by Martin and Ishida (1989) also showed that collagen fiber orientation was the best predictor of cortical bone tensile strength, while mineral density alone was a popr predictor. In the present study, although statistically significant correlations $(p<0.05)$ between the mineral density and the modulus were found in both trabecular and cortical specimens [Fig. 4(a)], the mineral density explains only $58 \%$ and $30 \%$ of variance in the modulus respectively. This suggests that the remaining large variance might be explained by the microstructural characteristics of each specimen.

Trabecular bone which is formed by surface remodeling contains angular segments, called 'trabecu- lar packets' (Jee, 1983). The segments are delineated by cement lines, and each segment has its own lamellae orientation. Therefore, we might expect a mosaic-like structure with more cement lines and variable lamellae orientation in trabecular bone tissue, which might have provided more stress risers, resulting in the lower modulus. This more segmented structure may also make trabecular bone tissue more isotropic than cortical bone tissue and may be a reason for the better correlation between modulus and mineral density in trabecular bone tissue.

As mentioned earlier, the mineral density of trabecular specimens was higher than that of cortical specimens. While this finding showed a result opposite to the observations of Gong et al. (1964) and Hodgskinson et al. (1989), where the mineral content of trabecular bone was lower than that of cortical bone, this could be partly explained by the remodeling characteristics along with the specimen preparation methods. Surface remodeling provides a newly formed bone (less mineralized) and osteoid on the surfaces of trabeculae, while older bone (higher mineralized) is in the center portions of trabeculae. Since most of the trabecular surfaces were removed during the specimen preparation procedures in the present study, our specimens may have been primarily composed of the highly mineralized portions of the trabeculac. On the other hand, the specimens in Gong's and Hodgskinson's studies included the less mineralized surfaces, perhaps resulting in a lower overall mineral density.

Another effort was made to investigate the effects of trabeculae orientation on the modulus within a localized trabecular bone volume. We hypothesized that vertically oriented trabeculae might have different modulus values from horizontally oriented trabeculae, because each trabeculae group might experience different mechanical environments, perhaps resulting in different microstructures. The data illustrated that the mean modulus of vertically oriented trabeculae was about $27.5 \%$ higher than that of horizontally oriented trabeculae. However, no statistically significant difference was found $(p>0.05)$. At this point, it is not possible to draw any conclusion about this result because of the very small sample size.

It should be noted that our trabecular tissue modulus (4.59 GPa) was obtained from specimens with an average height of $127 \mu \mathrm{m}$. If we assume that the trabecular tissue modulus has a size-dependency similar to that found for cortical bone, then the tissue moduli in bending might be expected to range from approximately 4 to $7 \mathrm{GPa}$ for typical trabecular thicknesses of $100-200 \mu \mathrm{m}$.

\section{The size-dependency of cortical bone modulus}

The effects of specimen size on the cortical bone modulus have not been studied extensively. Murray et al. (1984) tested three different sizes of cortical specimens $(t=0.1-2.5 \mathrm{~mm})$, and found that the modulus decreased with specimen thickness. No attempt 
was made to correlate the modulus and specimen size in their study. On the other hand, contradicting data have been reported by Yang and Lakes (1982), where modulus increased as specimens became smaller $(d=5.2-1.4 \mathrm{~mm})$. This study. however, was conducted on only large specimens, thus the application of this result to microspecimens may not be valid and may need to be tested separately.

In the present study, we examined the size-dependency of cortical bone modulus with both microspecimens ( $h<500 \mu \mathrm{m}$, five different sizes) and large specimens ( $h>500 \mu \mathrm{m}$, three different sizes), and found a significant correlation between the modulus and specimen size. One possible explanation for the size-dependency might be the presence of microstructural defects, such as lacunae, near the surfaces of the specimens (Rice et al., 1988). Since the size of these defects does not change with specimen size, as specimens become smaller the effects of the defects become more protound. It seems that these effects are not significant for the large specimens, but they gradually become significant as the specimen size approaches a microstructural level. The surface area to volume ratio has previously been proposed as a variable to explain the size-dependency of the modulus by Rice et al. (1988). A significant correlation was found between the modulus and surface area to volume ratio, and the relationship was summarized as a quadratic equation.

Although the elastic modulus or Young's modulus is considered an intrinsic matcrial property, a constant value regardless of specimen size, this assumes that the material is truly homogeneous. In fact, bone is not a homogeneous material. It consists of different constituent materials and possesses structural heterogeneity at every level of magnification. Even as test specimens are reduced in size in an eftort to approach some level of homogeneity, the previously insignificant microstructural characteristics become significant. The modulus of bone tissue determined even on a microstructural level may still represent a 'stiffness' rather than an intrinsic property of bone material. This aspect was previously described by Reilly and Burstein (1974) * . . . to some degree bone is a structure at all levels of organization including the molecular level, and this complex nature makes the size of the machined specimen an important variable to be considered in all tests. Because of the heterogeneity of bone, a working definition of the 'material' under test is needed'. Therefore, we believe that the direct comparisons between trabecular tissue modulus ( $\sim \mathrm{GPa})$ with large cortical bone modulus ( $\sim 15 \mathrm{GPa}$ ) could introduce a misunderstanding of the mechanical competence of both bone materials.

The tissue modulus values provide essential input data for finite element analyses of bone structure or bone-implant interfaces on a microstructural level (Hollister et al., 1990; Pedersen et al., 1990). Until microstructural models of bone include cement lines, lacunae, and other microstructural characteristics, the bone tissue 'stiffness' values which our data provide may be the most relevant to tissue moduli. Although, in tension or compression, the size-dependency may not be as serious as in bending. we believe that 'specimen size' should be considered as an important factor affecting the modulus on a microstructural level.

Acknowledgements-Support for this study was provided by a grant from National Institute of Health (AR31793, AR34399, AR20557) and the Whitaker Foundation.

\section{REFERENCES}

Ascenzi, A. and Bonucci, E. (1967) The tensile properties of single osteons. Anat. Rec. 158, 375-386.

Ascenzi, A. and Bonucci. E. (1968) The compressive properties of single osteons. Anat. Rec. 161. 377-392.

Ashman, R. B. and Rho, J. Y. (1988) Elastic modulus of irabecular bone material. J. Biomechanics 21, 177-181.

Brown, T. D. and Vrahas, M. S. (1984) The apparent elastic modulus of the juxtarticular subchondral bone of the (cmoral head. J. Orthop. Res. 2. 32-38.

Burstein, A. H.. Currey, J. D., Frankel, V. H. and Reilly, D. T. (1972) The ultimate propertics of bone tissue: the effects of yielding. J. Biomechanics 5, 35-44.

Currey. J. D. and Butler, G. (1975) The mechanical properties of bone tissue in children. J. Bone Jt Surg. 57A, 810-814.

Dempster, W. T. and Liddicoat. R. T. (1952) Compact bone as a non-isotropic material. Am. J. Anat. 91, 331-362.

Duncan, H., Jundt, J., Riddle. J. M., Pitchlord, W. and Christopherson, T. (1987) The tibial subehondral plate-a scanning electron mictoscopic study. J. Bone Ji SurH. 69A, 1212-1220.

Evans, F. G. and Lebow, M. (1951) Regional difterences in some of the physical properties of the human femur. J. appl. Physiol. 3, 563-572.

Feldkamp, L. A. and Jesion, G. (1986) 3D X-ray computed tomography. Review of Progress in Quantitative Nondestructive Evaluation (Edited by Thompson, D. O. and Chimenti, D. E.). Plenum Press, New York.

Frost, H. L. (1960) Presence of microscopic cracks in in vivo bone. Ilenry Ford Hosp. Bull. 3, 25-35.

Gung J. K., Arnold, J. S. and Cohn. S. H. (1964) Composition of trabecular and cortical bone. Anat. Rec. 149, 325-332.

Hodgskinson, H., Currey, J. D. and Evans, G. P. (1989) Hardness, an indicator of the mechanical competence of cancellous bone. J. Orthop. Res. 7, 754-758.

Hollister, S. J. Goldstein, S. A.. Jepsen, K. J. and Goulet, R. W. (1990) Continuum and microstructural stress morphology relationships for trabecular bone subject to controlled implant loads. Trans. 36th Orthop. Res. Soc. 15, 74.

Jee, W. S. S. (1983) The skeletal tissues. Histology: Cell and Tissue Biology (Edited by Weiss, L.), pp. 200-255. Elsevier, Amsterdam.

Ko, R. (1953) The tension test upon the compact substance of the long bones of human extremities. J. Kyoto Pref. Med. Univ. 53, 503-525.

Kuhn, J. L., Goldstein, S. A., Choi, K., London, M., Feldkamp, L. A. and Matthews, L. S. (1989) A comparison of the trabecular and cortical tissue moduli from human iliac crests. J. Orthop. Res. 7, 874-886.

Martin, R. B. and Ishida, J. (1989) The relative effects of collagen fiber orientation. porosity. density. and mineralization on bone strength. J. Biomechanics 22, 419-426.

Martin. R. B. and Pramanik. J. (1989) Effects of aspect ratio on the bending behavior of bone and wood. Proc. $13 \mathrm{th} \mathrm{Am}$. Soc. Biomechanics, pp. 22-23. Burlington, Vermont.

Mente. P. L. and Lewis. J. L. (1987) Young's modulus of trabecular bone tissue. Trans. 33rd Orthop. Res. Soc. 12, 49 
Murray, R. P. Hayes, W. C.. Edwards, W. T. and Harry. J. D. (1984) Mechanical properties of the subchondral plate and the metaphyseal shell. Trans. 30th Orthop. Res. Soc. 9, 197.

Pedersen. D. R., Brown. T. D. and Brand, R. A. (1990) Bone ingrowth stress distributions within a novel prosthesis anchorage layer. Trans. 36th Orthop. Res. Soc. 15, 205.

Reilly. D. T. and Burstein. A. H. (1974) The mechanical properties of cortical bone. J. Bone Jt Surg. S6A. 1001-1022.

Reilly. D. T. and Burstein. A. H. (1975) The elastic and ultimate properties of compact bone tissue. J. Biomechanics 8. 393-405.

Reilly, D. T. Burstein, A. H. and Frankel, V. H. (1974) The elastic modulus for bone. J. Biomechanics 7, 271-275.

Rice, J. C., Cowin, S. C. and Bowman, J. A. (1988) On the dependence of the elasticity and strength of cancellous bone on apparent density. J. Biomechanics 21, 155-168.

Runkle, J. C. and Pugh. J. W. (1975) The micromechanics of cancellous bone-1i. Determination of the elastic modulus of individual trabeculae by buckling analysis. Bull. Hosp. Jt Dis. 36, 2-10.

Ryan, S. D. and Williams, J. L. (1989) Tensile testing of rodlike trabeculae excised from bovine femoral bone. J. Biomechanics 22, 351-355.

Sedlin. E. D. (1965) A rheological model for cortical bone. A study of the physical properties of human femoral samples.
Acta orthop. scand. Suppl. 83.

Sedlin, E. D. and Hirsch, C. (1966) Factors affecting the determination of the physical properties of femoral cortical bone. Acte orthop. scand. 37. 29-48.

Simkin. A. and Robin, G. (1973) The mechanical testing of bone in bending. $J$. Biomechanics 6, 31-39.

Tamopolskii. Iu. M. and Kincis. T. (1985) Static Test Methods for Composites, pp. 221-252. Van Nostrand Reinhold. New York.

Timoshenko, S. P. and Goodier, J. N. (1987) The Theory of Elasticity. pp. 113-122. McGraw-Hill, New York.

Townsend. P. R., Rose. R. M. and Radin, E. L. (1975) Buckling studies of single human trabeculae. J. Biomechanics 8, 199-201.

Weaver, J. K. (1966) The microscopic hardness of bone. J. Bone Jt Surg. 48A. 273-288.

Williams, J. L. and Lewis, J. L. (1982) Properties of an anisotropic model of cancellous bone from the proximal tibial epiphysis. J. biomech. Engng 104, 50-56.

Yamada, H. (1970) Strength of Biolngical Materials (Edited by F. G. Evans), pp. 19-37. Williams and Wilkins. Baltimore.

Yang. J. F. C. and Lakes, R. S. (1982) Experimental study of micropolar and couple stress elisticity in compact bone in bending. J. Biomechunics 15, 91-98. 